\title{
Rehabilitación y adaptación de la arquitectura para espectáculos en Extremadura
}

\author{
Restoration and adaptation of the scenic architecture \\ in Extremadura \\ LAURA FERNÁNDEZ ROJO \\ Becaria FPI Gobierno de Extremadura. Universidad de Extremadura \\ lfdezrojo@gmail.com
}

Recibido: $12 / 10 / 2014$

Aceptado: 08/04/2015

\begin{abstract}
Resumen
La intervención que presentamos está orientada a evaluar de forma crítica el proceso de conservación, rehabilitación y adaptación de diversos edificios como espacios para espectáculos, tema que nos permite estudiar los usos posibles de un importante conjunto de edificaciones de la cultura del ocio de todos nosotros. Veremos cómo han subsistido hasta nuestros días y las actuaciones que han tenido que hacerse sobre los mismos, el criterio que se ha seguido, si éste ha respetado el original o no y su actualización al siglo XXI, las reformas pertinentes y su función actual. En este estudio reflexionamos sobre la evolución de los espacios escénicos de Extremadura, para demostrar que estos locales son necesarios y que se sigue abogando por su construcción y mantenimiento. Quisiéramos reivindicar el patrimonio extremeño en el ámbito que concierne al ocio del espectador, es decir, valorándolo a través de las obras escogidas.
\end{abstract}

Palabras clave

Arquitectura, cine, teatro, restauración, Extremadura. 


\begin{abstract}
The intervention that we present take aim at the critical evaluation of the process of conservation, refurbishment and adaptation of diverse buildings as performance spaces, theme that allows us to study the possible uses of an important set of constructions of leisure culture of us all. We will see how they have survived to this day and the interventions made on them, the guideline to follow, if it has respected the original or not and their updates in the XXI century, relevant reforms and their current role. In this study we reflect on the evolution of scenic buildings Extremadura, to show that these premises are necessary and which continues to advocate for their construction and maintenance. We wanted to claim the heritage of Extremadura in the field concerning the entertainment of the viewer, in other words, valuing through selected works.
\end{abstract}

\title{
Keywords
}

Architecture, cinema, theatre, restoration, Extremadura.

Referencia normalizada: FERNÁNDEZ ROJO, L. (2015): “Rehabilitación y adaptación de la arquitectura para espectáculos en Extremadura". Arte y Ciudad. Revista de Investigación, no 7 (abril), págs. 7-26. Madrid. Grupo de Investigación Arte, Arquitectura y Comunicación en la Ciudad Contemporánea, Universidad Complutense de Madrid.

Sumario: 1.- Arquitectura escénica en Estremadura. 1.1.- Central Cinema, Azuaga (Badajoz). 1.2.- Círculo Pacense, Badajoz. 1.3.- Teatro Cine San Vicente, Fuentes de León (Badajoz). 1.4.- Auditorio La Merced, Llerena (Badajoz). 1.5.- Teatro Municipal, Zafra (Badajoz). 1.6.- Teatro del Mercado, Navalmoral de la Mata (Cáceres). 1.7.- Teatro Gabriel y Galán, Trujillo (Cáceres). 2.- Conclusiones: líneas de conservación en la arquitectura para espectáculos. 3.- Bibliografía.

\section{Arquitectura escénica en Extremadura.}

El hecho creciente de la desaparición de edificios dedicados a espectáculos tanto en ciudades como en pequeñas localidades, hace necesario llevar a cabo estudios sobre esta tipología arquitectónica. Y quizás sea esta la razón que motiva la rehabilitación de edificios en desuso como espacios escénicos, aunque también por la crisis del sector teatral y cinematográfico muchos de estos edificios se están rehabilitando como sedes culturales, que al ser más funcionales garantizan la perdurabilidad del inmueble. 
La adecuación de uso de estos edificios se mueve entre dos tendencias: la que busca la rentabilidad del bien y la que intenta garantizar su perdurabilidad. En nuestra opinión, de las soluciones adoptadas en esta tipología arquitectónica la idónea, porque es la más conservacionista con la obra, es convertir estos inmuebles en contenedores culturales pues garantiza tanto su rentabilidad como su preservación: al ofrecer un amplio programa cultural se favorece el uso del cine o teatro preservando indirectamente el bien, y no supone grandes obras en el edificio sino en la mayoría de los casos es suficiente con una restructuración interior.

\subsection{Central Cinema, Azuaga (Badajoz).}

En Extremadura contamos con teatros de gran valor histórico artístico, como el Central Cinema de Azuaga (Badajoz), que entonces se le conocía como Teatro Espino y fue construido por Manuel Carrascal, tío abuelo de la pintora Mayte Spínola ${ }^{1}$. Es un edificio de estilo modernista construido en el primer tercio del siglo XX. El local principal se levantó como teatro pero funcionaba también como sala de fiestas, cine y local para diversas actividades culturales. En su etapa final fue un salón recreativo².

El inmueble está situado en el centro histórico de Azuaga, junto a la Plaza del Cristo y muy cerca del Teatro Cine Capitol. Las obras de rehabilitación del Central Cinema constaron de tres fases: la primera en 1995 según proyecto de los arquitectos José Manuel Jaureguibieitia Olalde y Arsenio Rica Cámara; la segunda en 2002 de José Manuel Jaureguibieitia Olalde y la última en 2008 de la aparejadora Nuria Barragán del Puerto.

Ante el desuso del edificio, las autoridades lo adquieren y en 1995 adecuan la planta de arriba como teatro y Museo de Arte Contemporáneo, y el sótano como Museo Etnográfico. Se optó por la solución de Museo Etnográfico porque Extremadura fue de las primeras que comenzaron a ahondar en los estudios de etnografía, y esta tipología museística está muy arraigada entre los extremeños.

\footnotetext{
${ }^{1}$ Información aportada por Mayte Spínola: Heredó el local (teatro y bodega) el padre de la pintora, Máximo Spínola. El piso de arriba funcionó como cine y el de abajo como “Bodegas de Máximo Spínola" hasta que murió el propietario. Sus hijos, Mayte, José Antonio, Conchita y Carmen, vendieron todo el edificio al Ayuntamiento de Azuaga en 1988.

${ }^{2}$ Ayuntamiento de Azuaga, Servicio de Obras, Jaureguibeitia, J.M. y Rica, A., Anteproyecto de rehabilitación y adaptación para museo, Azuaga, 1995.
} 


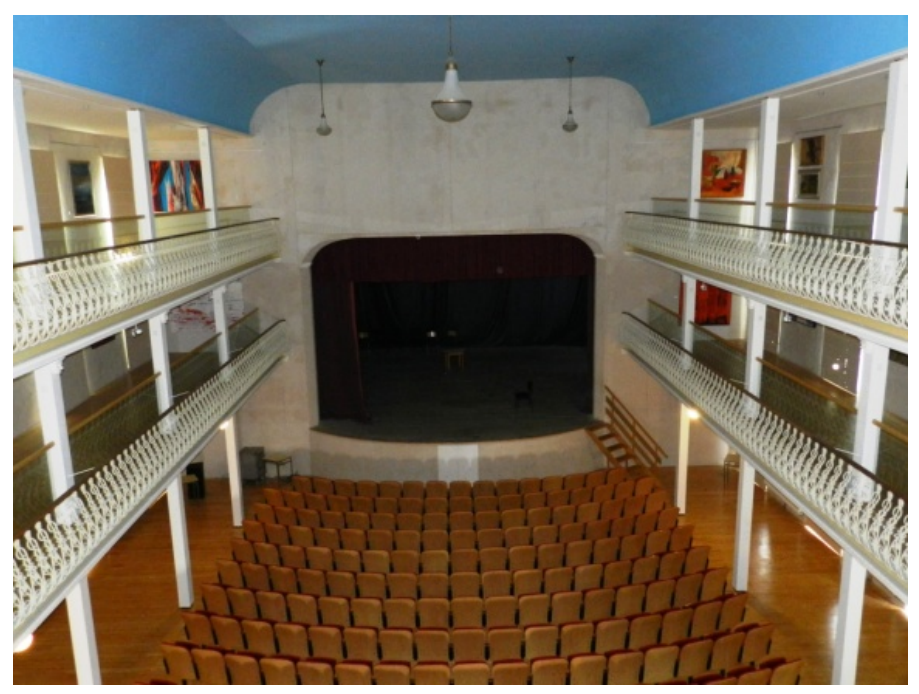

Fig. 1.- Central Cinema de Azuaga (Badajoz) (Fotografía: Fernández Rojo, L.)

Pero no será la única intervención en el edificio: en 2008 se rehabilita de nuevo el inmueble para completar las fases anteriores, afectando sobre todo al Museo Etnográfico, y al año siguiente se acondiciona el Museo de Arte Contemporáneo (Barragán del Puerto, 2009). La intervención en el sótano ha respetado el espacio de la bodega; de hecho, se ha evitado picar y enlucir paredes, se han mantenido conos, ladronas, toneles, etc., buscando preservar el ambiente original. Además, se han reutilizado los elementos que estaban en buen estado de conservación como su primitiva puerta de madera o los tablones y el cristal en los entrepaños de los pilares, e incluso mantiene su estructura de arcadas originales de ladrillo visto para atestiguar cómo era y demostrar que se ha modificado, facilitando el análisis de la antigua bodega habilitada como museo.

El teatro ha sido restaurado conservando su estética original, se han reproducido escayolas y bronces, se ha mantenido la estructura y las formas, la iluminación, etc. Es fiel a la línea de intervención en la bodega, pues, a pesar de que se rehabilita con tendencias arquitectónicas actuales, se diferencia entre lo intervenido y lo anterior tal como vemos en su fachada al mostrar en su acabado los materiales constructivos originales que eran la piedra y el ladrillo y solerías exteriores a base de piedra caliza. Otro ejemplo sería que al tener que sustituir un elemento se cambia por otro nuevo pero similar al anterior; prueba de ello es su falso techo de escayola. 
A pesar de tres proyectos de rehabilitación en el Central Cinema, quedan cuestiones sin resolver. La primera de ellas es que no se ha establecido el circuito continuo desde los palcos al escenario, lo cual hubiera supuesto un mayor espacio para la exposición de las obras al aumentar el recorrido por el edificio. A este respecto José Manuel Jaureguibieitia nos asegura que la estructura estaba hecha, pero por falta de presupuesto no se llevó a cabo. Y la segunda es que al mismo tiempo que se realizaba la rehabilitación del Cine Teatro Capitol también se desarrollaba la del Central Cinema; por este motivo el peine de madera del primero se pretendía trasladar al segundo, pero ni el Capitol tiene concha acústica ni el Central Cinema tiene peine de madera.

No se han salvado estas faltas, y no solo eso, sino que en las dos últimas intervenciones llevadas a cabo por la municipalidad no se ha tenido en cuenta el primer proyecto. Pues en la primera rehabilitación se olvida por completo el teatro y las carencias que tiene y se centra la intervención en la bodega, y en la segunda no se tiene en cuenta la función original del teatro, otorgándole un nuevo uso como museo.

El Central Cinema es un teatro con un gran aforo que tiene incluso palcos, lo que no es habitual en los teatros de Extremadura: la mayoría de ellos solo tienen patio de butacas y anfiteatro. Así, este espacio escénico singular se está desaprovechando inculcándole una función distinta a la original, la de Museo de Arte Contemporáneo. Aunque en los proyectos se pretende conciliar ambos usos (teatral y museístico), esto no se lleva a la práctica, y la realidad es que la localidad podía contar con dos espacios dedicados al cine y al teatro y solo se utiliza uno de ellos.

\subsection{Círculo Pacense, Badajoz.}

Los dos edificios que componen el Círculo Pacense se encuentran ubicados en pleno casco antiguo de Badajoz dentro del recinto intramuros de la muralla Vauban de los siglos XVII y XVIII. Ventura Vaca Parrillas fue el arquitecto autor del proyecto en 1914 y Rodolfo Martínez González la ampliación en 1931. Eduardo Escudero Morcillo dirigió la reforma realizada en 1972 y Javier Teijeiro Fuentes efectúa en 1998 un informe técnico sobre el valor arquitectónico de los inmuebles, dando lugar al proyecto de rehabilitación llevado a cabo en 2002. 
En 1913 se le conocía como Círculo Pacense; tras la Guerra Civil, la Sociedad Centro Obrero se convirtió en una sociedad privada y pasó a manos de la Organización Sindical de Educación y Descanso, y el 21 de diciembre de 1939 Ramón Espino Méndez, como arrendatario del Centro Obrero, solicita licencia de obra para la adaptación del local a cinematógrafo, entonces pasaría a denominarse Cine Pacense hasta principios de los $90^{3}$.

Las actuaciones en el edificio no han terminado porque quedan áreas por rehabilitar, la planta baja es la que mejor está, pero las superiores sufren graves desperfectos. Por ejemplo en la primera planta, donde está la biblioteca y sobre todo en la segunda altura, hay humedad, maderas carcomidas y techos con filtraciones. Y más aún la segunda planta en la que hay estancias prácticamente en ruina.

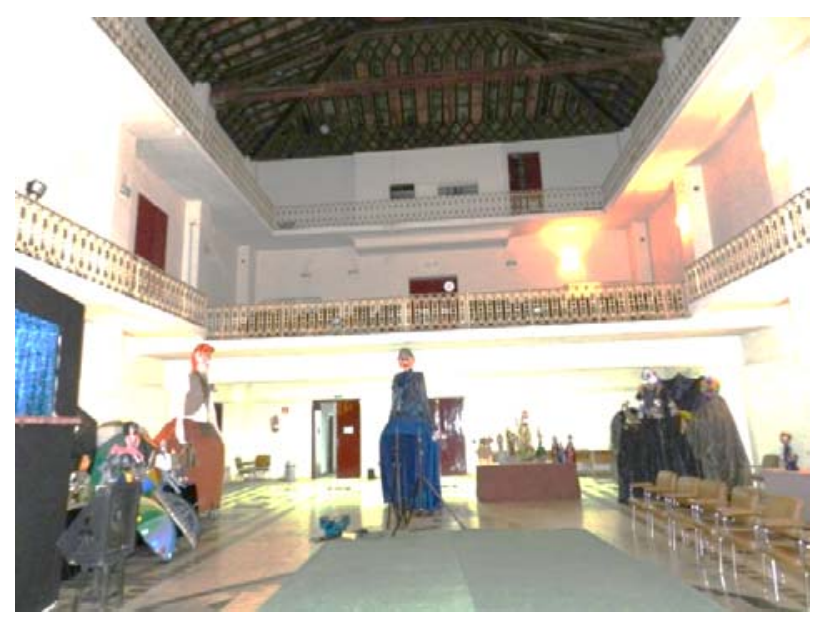

Fig. 2.- Círculo Pacense de Badajoz (Fotografía: Fernández Rojo, L.)

El arquitecto Javier Teijeiro llevó a cabo un estudio arquitectónico en el que valoraba el estado de conservación del centro. En él se destaca que la parte superior y la ampliación de 1931 son las más deterioradas, ya que hay filtraciones que estropean la madera y la pintura. La cubierta de madera en forma de artesa del salón principal corre peligro. En ella son cada vez más visibles los desperfectos causados por las filtraciones de agua (Teijeiro Fuentes, 1998). El principal problema en este caso es la cuestión económica: no hay fondos para hacer frente a las intervenciones que necesita. De hecho, para fina-

${ }^{3}$ Archivo Histórico Municipal de Badajoz, Secc. Vías y Obras, legajo 111, expediente 827, 1939. 
lizar la adecuación del lugar, que duró ocho años, la sociedad inició una campaña de captación de socios para hacer frente económicamente a las obras. Pero el presupuesto fue insuficiente y solo se rehabilitó la planta baja: $2.500 \mathrm{~m}^{2}$. A pesar de no estar terminado, los socios consiguieron la licencia de apertura, aunque únicamente para el bajo.

Pese a que se ha eliminado la amenaza de ruina que asolaba al inmueble, su actividad está limitada por las críticas condiciones en las que se encuentra. La recuperación total de este centro depende de la rehabilitación integral de su patrimonio, no habiendo fecha para esto. Aun así tras la intervención de 1998 tiene una amplia oferta cultural, desde partidos de hockey o baloncesto, patinaje, clases de portugués, teatro o incluso veladas pugilísticas. Además facilitan su local de forma gratuita a diversos colectivos de la ciudad para que puedan desarrollar sus proyectos. Por lo que actualmente es una sala multifuncional como corresponde al enfoque moderno que caracteriza a la Sociedad y pese a su estado de conservación, ha conseguido poner en uso un edificio en ruina tal y como planteaba Javier Teijeiro en su proyecto.

\subsection{Teatro Cine San Vicente, Fuentes de León (Badajoz).}

Se trata de un edificio exento, situado en la zona noroeste de Fuentes de León, con acceso principal por la Avenida de Portugal y con otros por las calles anexas. El Teatro-Cine San Vicente es del siglo XIX o comienzos del XX, su estilo es Neomudéjar y de especial interés son los plafones modernistas. Está catalogado como edificio de valor histórico artístico categoría 1 grado 2, grupo que comprende las edificaciones de valor histórico y arquitectónico que por su calidad, antigüedad, escasez o rareza deben ser conservados en todas sus características, tanto exteriores como interiores, permitiéndose obras de consolidación, conservación, restauración y reforma restringida, siempre y cuando no afecte esta última a elementos significativos. Las dos obras de restauración que se realizan son proyectadas por sendas arquitectas técnicas, Agustina Redondo Rosario en 2006 y Elia Barco Guerrero en 2011.

El primer proyecto se centra en reparar la cubierta, pues su estado de conservación era bastante deficiente, con numerosas goteras y desprendimientos de gran parte del falso techo ${ }^{4}$. Y el segundo está encaminado a mejorar la accesibili-

${ }^{4}$ Archivo municipal de Fuentes de León, expediente PR-398-SP-IF-1-06, Redondo Rosario (2006).

Arte y Ciudad - Revista de Investigación

$N^{\circ} 7$ - Abril de 2015 
dad del inmueble, pues las rampas de acceso tenían una pendiente excesiva, las escaleras exteriores de acceso al bar estaban en malas condiciones de conservación y también se encontraba en mal estado el pavimento del patio exterior ${ }^{5}$.

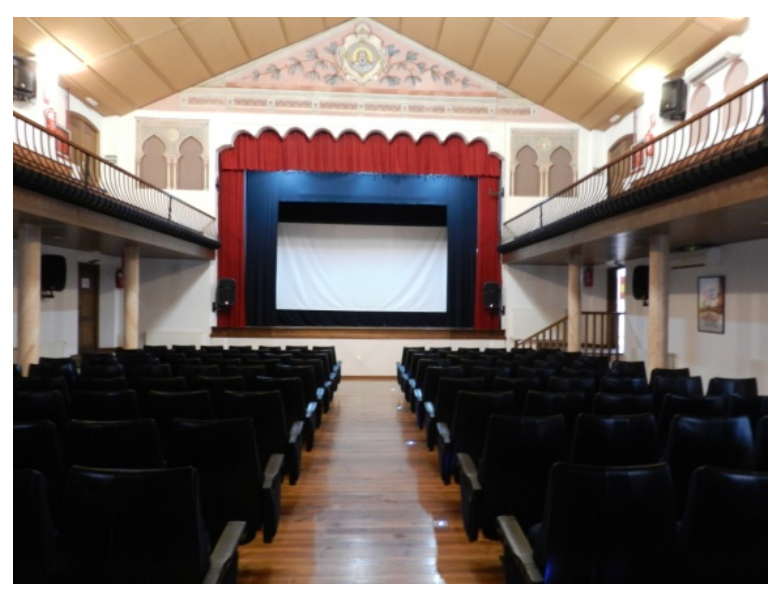

Fig. 3.- Patio de butacas del Teatro Cine San Vicente de Fuentes de León (Badajoz) (Fotografía: Fernández Rojo, L.)

La intervención en el teatro ha respetado la traza primitiva del inmueble, ya que era indispensable preservarla, pues en Extremadura no hay otro teatro neomudéjar, y sin ella no se podría hacer una lectura biográfica del edificio. Las obras, que han durado tres años, han ido encaminadas a hacer el inmueble más accesible para garantizar su uso. Por este motivo, las intervenciones se han centrado en el teatro propiamente dicho al ser el espacio principal y en los accesos a este. Además, se han aprovechado las estancias del teatro, para otorgarles una nueva función cultural más acorde con el carácter lúdico del edificio. De manera que ya ha desaparecido su antiguo uso como casa de beneficencia, pues no tenía sentido mantenerlo cuando ya no es la Hermandad quien lo gestiona sino la municipalidad. Como consecuencia del cambio de titularidad, la antigua casa estaba en desuso, rehabilitándose como Asociación de Mujeres Rurales. Los antiguos vestuarios ya no eran necesarios porque se habían habilitado nuevos, por lo que se convirtieron en una estancia dedicada a fines lúdicos y recreativos.

Dichas obras van acorde con los proyectos de transformación de estos espacios escénicos en centro culturales. La realidad es que si se mantuvieran para una sola función sería condenarlos a un futuro cierre por no utilizarse el establecimiento.

${ }^{5}$ Ibídem, expediente 2011-170, Barco (2011). 
Por ser un inmueble tan antiguo, carecía de un equipamiento actualizado; ni siquiera tenía un sistema contraincendios ni luces de señalización de emergencia, y para su correcto funcionamiento como cine era necesaria una nueva pantalla de proyección. Además, hay elementos que no se rehabilitaron, como la cabina de proyección y los camerinos, y otros que sí pero que necesitan otra intervención, como el aislante de la cubierta. Las obras han sido coherentes con su traza primitiva y han actualizado el edificio al otorgarle un nuevo uso más acorde a nuestro tiempo, pero las intervenciones no han sido suficientes para garantizar la perdurabilidad y el uso del teatro.

\subsection{Auditorio La Merced, Llerena (Badajoz).}

El Centro Cultural La Merced está dentro del casco histórico declarado como Bien de Interés Cultural, según Decreto 3302/1966 de 29 de diciembre, por el que se declara conjunto histórico artístico la ciudad de Llerena. Sigue el modelo de las iglesias jesuíticas proyectadas por el arquitecto Giacomo Barozzi da Vignola (1507-1573), la fachada principal se presenta lisa, volúmenes claros, con un marcado ritmo ascendente del crucero que se remata con un tambor, una linterna y unos potentes pináculos. La espadaña realizada en ladrillo sería de época posterior. En la actualidad, se utiliza como teatro o salón de actos $^{6}$. Los autores de la transformación de iglesia a teatro fueron los arquitectos Luis de Aréchaga Sánchez Pascual en 1982 y Juan Antonio López Galíndez en 1986.

Es una iglesia barroca construida en 1630, tiene planta de cruz latina con cimborrio en el crucero, capillas laterales en el brazo mayor y tribunas. Las capillas laterales en la actualidad se usan como salas de exposiciones y son en total tres pequeñas salas que se comunican entre sí, de planta cuadrangular, cubiertas por bóvedas de arista, dotadas de rieles y focos alógenos. La planta de la iglesia es la habitual en los templos de la Compañía de Jesús: de cruz latina con transepto poco marcado, nave única con bóveda de medio cañón con lunetos, capillas entre contrafuertes, y cúpula con pechinas sobre el crucero.

En la primera actuación se plantea transformar la Iglesia en Centro Cultural para utilizarlo como salón de actos, para conferencias y conciertos, sala de expo-

${ }^{6}$ Ayuntamiento de Llerena, Servicio de Obras, Granada Hernandez Pachón (2012). 
siciones, biblioteca y museo artesanal7 ${ }^{7}$. Esta intervención se amplió con el proyecto dirigido por Juan Antonio Galíndez, que se centró en los problemas acústicos $\mathrm{y}$, finalmente se acometen una serie de obras para mejorar la accesibilidad.

La rehabilitación ha respetado en la medida de lo posible el edificio que fue reutilizado como colegio, iglesia y almacén de grano. No sabemos a ciencia cierta cómo era originalmente, sólo que ha sido fiel al estado previo del edificio antes de las reformas citadas. De hecho, las intervenciones han sido las menos posibles, teniendo en cuenta que modifica la función del edificio drásticamente prescindiendo de su carácter litúrgico.

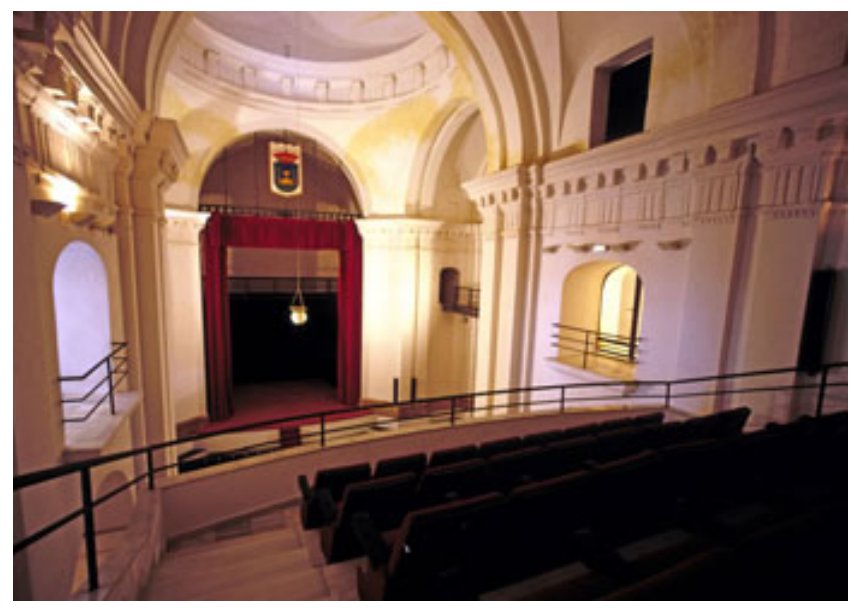

Fig. 4.- Centro Cultural La Merced, Llerena (Badajoz) (Fotografía: Fernández Rojo, L.)

Exteriormente las obras se limitan a corregir los problemas de humedad sin modificar ningún elemento, salvo la cubierta que, debido a su deterioro, debía cambiarse y lógicamente se opta por una estructura moderna que no desentonara con la estética del convento.

Sin embargo, las actuaciones en su interior han sido en mayor número por el estado de ruina del teatro, pero por suerte estructuralmente su estado de conservación era bueno, por lo que tales obras no han sido muy agresivas con el inmueble. Nos parece acertado que se haya optado por aprovechar los espacios y que las obras sean mínimas, pues podrían haber hecho palcos, patio de butacas, camerinos, etc. Pero en vez de eso se han aprovechado las propias

${ }^{7}$ Archivo Histórico Municipal de Llerena, carpeta núm. 5, legajo 1166, De Arechaga (1982). 
estancias del convento de manera que las tribunas se utilizan como palcos, el patio de butacas y el anfiteatro son desmontables, el escenario es una estructura independiente que se ha encajado en el presbiterio, etc., facilitando que sea un espacio reversible y multiusos ${ }^{8}$.

Un edificio no debe desvincularse de su primitiva función porque se descontextualiza, pero antes de que el edificio caiga en desuso es una buena opción rehabilitarlo como teatro, siempre que las intervenciones para tal fin sean las mínimas e imprescindibles para garantizar la seguridad y perdurabilidad de la obra. Lo que sí hemos observado es que en estos edificios tan abiertos y espaciosos y con grandes cúpulas, como el Centro Cultural La Merced, es difícil conseguir una buena acústica. Esta es una asignatura pendiente en La Merced y que es vital por su carácter escénico, por lo que si no se soluciona esta cuestión quizás convendría darle un uso cultural como sala de exposiciones, talleres, etc., que son actividades en las que el factor acústico no es tan importante como en las obras de teatro o conciertos, que es su principal reclamo cultural en la actualidad.

\subsection{Teatro Municipal, Zafra (Badajoz).}

La parcela de titularidad municipal se encuentra al lado de la ronda que envuelve extramuros la ciudad histórica desde su lado occidental. El solar, irregular y con una marcada pendiente, estaba prácticamente delimitado por las traseras de diversos corrales y viviendas tradicionales de particulares y el antiguo inmueble lo constituían dos edificaciones: los números 22 y 24 de la calle Fernando Moreno Márquez. La adaptación como teatro la realiza el arquitecto Enrique Krahe Marina en 2005.

Desde el punto de vista artístico, el Teatro Municipal Zafra es una joya arquitectónica sin parangón en Extremadura, que acerca las vanguardias europeas a la arquitectura para espectáculos. El edificio es una verdadera obra de arte en sí, pues es contenedor de diversas manifestaciones artísticas desde la pintura, la escultura, posibles performances y, cómo no, el teatro. De hecho, por su valor artístico y arquitectónico ha recibido el Premio Lamp Lighting Solutions 10' y el Premio Lamp, y ha sido nominado para los premios de la

${ }^{8}$ Servicio de Obras y Proyectos, Consejería de Cultura y Turismo, Gobierno de Extremadura, Aréchaga (1982). 
Asociación Internacional de Diseñadores de Iluminación (IALD) y para los premios Mies Van der Rohe. El día 25 de noviembre se falló el Premio de Arquitectura del Colegio Oficial de Arquitectos de Extremadura en su edición de 2013. A la convocatoria se presentaron veinte obras realizadas en Extremadura entre los años 2008 y 2013, y la obra a la que por unanimidad se otorgó el premio fue la del Teatro Municipal de Zafra.

En la parcela del Teatro Municipal diferenciamos tres volúmenes, el volumen principal o teatro propiamente dicho (que contiene la caja del escenario, sala principal y una sala de ensayos) y dos edificaciones auxiliares con elementos vernáculos, como son bóvedas tabicadas y muros de mampostería de pizarra.

El entorno tiene una serie de plazas con pequeños jardines adosados al perímetro de la parcela. Los pavimentos que delimitan tales jardines son de calzada portuguesa y de hormigón. La misma calzada portuguesa que pavimenta el exterior se extiende en el vestíbulo del teatro y en los espacios accesorios del mismo. Una continuidad parecida se da entre el solado del escenario y el linóleo negro del patio de butacas, donde fieltros naturales que rememoran a las ovejas que antes pastaban en la parcela revisten los paramentos de su interior. Siguiendo con la utilización de materiales naturales y propiamente autóctonos, no hay que olvidar el aplacado de corcho que recubre la sala de ensayos.

En los aseos públicos, los espejos se convierten en camerinos, donde los espectadores se pueden caracterizar de modo similar al que lo harían los actores con algunos complementos dibujados en los espejos. En la sala principal, los cinco colores que tapizan las 361 butacas se combinan de modo que, percibidas desde el escenario, constituyen un gran ojo. Con este recurso, el arquitecto pretende que el escenario esté provisto siempre de un ojo pendiente de él.

El escenario se puede convertir en un espacio al aire libre que se abre por detrás al patio trasero, pavimentado por un tablero de ajedrez a escala humana rindiendo tributo al clérigo local Ruy López (campeón del mundo entre 1570 y 1575). El juego llega a su última escala, pues el espectador es un peón más en este tablero desde el que observa la obra de teatro que se desarrolla en el escenario (Krahe Marina, 2005).

Las obras acometidas le han otorgado un nuevo carácter cultural y han conseguido reavivar un área rural en desuso y en penoso estado de conservación, 
todo ello gracias a que se ha ligado el teatro con el núcleo urbano, haciéndole partícipe de la ciudad de la que -hasta ese momento- estaba desvinculado. Debemos destacar que la intervención no ha querido borrar el pasado del edificio, pues reutiliza locales preexistentes: deja la huella de las obras demolidas para que se pueda hacer una idea de cómo era anteriormente y como materiales utiliza los autóctonos, e incluso hace hincapié en recordar su antigua función como tenería ya que sus dos estancias principales, vestíbulo y sala principal, están revestidas de lana.

Fig. 5.- Teatro Municipal de Zafra (Badajoz) (Fotografía: Fernández Rojo, L.)

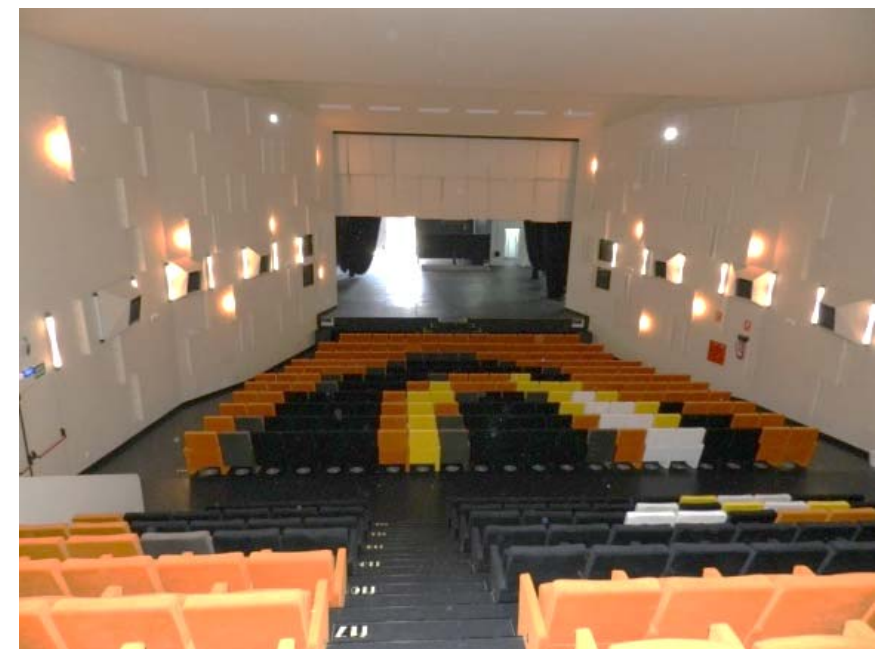

Por tanto, a pesar de ser un edificio totalmente innovador que nada tiene que ver con la obra que ocupaba el solar anteriormente, podemos hacer una lectura que nos lleve a deducir su anterior configuración y función. Podemos concluir que el proyecto de rehabilitación es coherente y acorde al momento en el que se ejecuta la intervención.

\subsection{Teatro del Mercado, Navalmoral de la Mata (Cáceres).}

El Teatro del Mercado de Navalmoral de la Mata está ubicado en la zona sur de la población, un poco apartado del centro, entre las calles Joaquín Alcalde, Mateo y Travesía Vázquez. Es un caso particular, pues no se trata de un cine o un teatro que vaya a someterse a una rehabilitación, sino que fue un mercado de abastos que se reforma como teatro, según proyecto de la arquitecta Matilde Peralta del Amo en 2007. 
El inmueble estaba constituido por dos naves que se unían en forma de L y el atrio de acceso con las zonas de almacenamiento. Las naves del mercado tenían una estructura basilical, siendo la central la de mayor altura, y las laterales presentaban en su testero un óculo circular en la pared alta, situándose en una de ellas muelle de carga y descarga (Peralta del Amo, 2007).

En Extremadura contamos con otro ejemplo similar, fue la plaza ocupada por el antiguo mercado de abastos de Montijo que se demolió para construir un teatro de nueva planta. Por lo que es una práctica habitual convertir estos edificios que están en desuso o desfasados en inmuebles culturales, ya que la mayoría han sido desplazados por las grandes superficies comerciales. Con esta práctica se está intentando recuperar la oferta cultural en diversos municipios, no concibiendo estos inmuebles únicamente como cines o teatros sino como sedes culturales para garantizar su uso.

Aparentemente, del antiguo mercado sólo cambian las cubiertas, pero se construye un nuevo edificio dentro del antiguo, es decir, que esta intervención no respeta el edificio original. Puede que se haya intentado preservar la fisonomía de su planta y parte de la estructura de la obra, pero el resultado es un edificio que nada tiene que ver con el antiguo mercado de abastos, ni los volúmenes proyectados, ni sus rasgos decorativos, pues ahora exteriormente es un bloque de hormigón cuando antes estaba pintado de blanco y ocre. Ni siquiera su funcionalidad ha permanecido vigente.

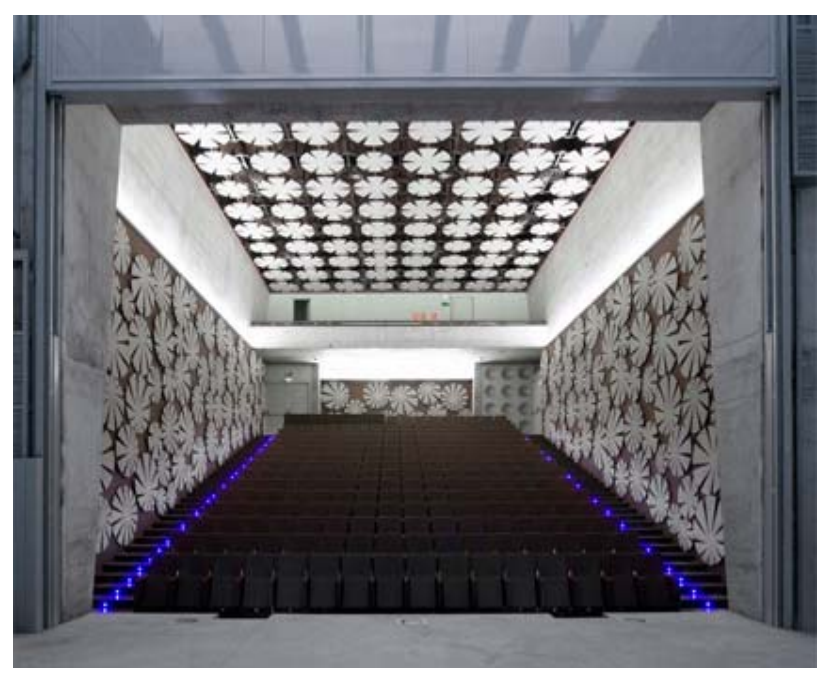

Fig. 6.- Teatro del Mercado, Navalmoral de la Mata (Cáceres) (Foto: Fernández Rojo, L.) 
Está constituido por tres volúmenes geométricos angulares y de diferentes alturas revestidos de un material cerámico gris oscuro. Estos volúmenes son prácticamente iguales salvo por su altura y en ellos permanecen las texturas de los moldes de madera que se emplearon para dar forma al material, que es hormigón. La convivencia entre lo viejo y lo nuevo en este caso es inexistente, lo que es razonable porque su fin no es el mismo y para su adaptación era necesario llevar muchas reformas y variaciones para que el local se actualizara a su nueva función.

El resultado es un teatro en cuya fisonomía destaca su juego de volúmenes y la torre escénica de 15 metros de altura, que en un principio iba a estar cubierta con motivos florales que hubieran reflejado la luz de forma especial según ésta incidiera en la torre.

\subsection{Teatro Gabriel y Galán, Trujillo (Cáceres).}

El Teatro Gabriel y Galán se encuentra en un lugar estratégico de Trujillo ya que conecta con el centro histórico y la plaza mayor, accediendo fácilmente a todos los lugares de la ciudad. El conjunto urbano fue declarado como Bien de Interés Cultural en 1962.

Ubicado en el Palacio Juan Pizarro de Aragón, su interior ha sido alterado por numerosas reformas en el siglo XX, entre las que podemos destacar la efectuada por el arquitecto José López Munera en 1929; en 1977 y 1980 las reformas las proyecta Dionisio Hernández Gil; en 1985 Carlos Baztán Lacasa; en 1986 José Ramón Zorita Carrero dirige la más profunda rehabilitación y en 2009 Studiodata transforma su jardín en plaza pública.

El primer proyecto de restauración del arquitecto Dionisio Hernández Gil consiste en nuevos forjados, cubiertas y pavimentos. En el segundo se encarga de la planta alta del palacio, en él se elimina gran parte de los aditamentos decimonónicos otorgándole un aspecto moderno al teatro.

Carlos Baztán Lacasa realiza la rehabilitación por el trámite de emergencia, ya que su estado de conservación era aceptable en términos generales pero en la zona de la galería de la fachada principal la ausencia de cubierta había deteriorado gravemente las bóvedas, hasta el extremo de suponer un peligro por posibles desprendimientos. Pero se hizo necesaria otra intervención des- 
pués, pues aunque el inmueble estaba parcialmente ocupado y dispuesto para su uso, su estado de conservación era lamentable encontrándose en ruina total por la pérdida de elementos de cubierta y forjados, y sólo en la zona central se mantenían los muros del primitivo teatro. Es por ello que en 1986 la Consejería de Educación y Cultura de la Junta de Extremadura encarga el proyecto para para ser utilizado como centro cultural al arquitecto José Ramón Zorita Carrero. Y su última intervención es la remodelación del jardín en 2009 por el arquitecto Graciliano Berrocal, que forma parte del equipo de arquitectura Studioata.

La intervención del arquitecto Zorita Carrero intenta ser lo más fiel posible con la primitiva sala, pues tiene una atractiva traza modernista que hay que mantener para conocer la historia del edificio. Hacerla desaparecer impediría su lectura biográfica.

Además, las actuaciones desarrolladas irían acorde con la idea de concebir el inmueble como un centro cultural, pues los teatros en estas pequeñas localidades han quedado relegados a un último plan al prescindirse de grandes escenarios y tramoya clásica porque se tiende a una participación del espectador o a pequeños escenarios. Por este motivo, concebirlos para una sola función sería condenarlos a un futuro cierre por el desuso del establecimiento 9 .

Aunque consideramos oportunas las actuaciones que se llevaron a cabo en los años ochenta, a día de hoy las deficiencias del Teatro Gabriel y Galán son evidentes. De hecho, las lleva sufriendo años y sobre todo afectan a la confortabilidad, pues si bien hay un sistema de calefacción los espectadores pasan frío en las butacas, hay filtraciones e incluso pudimos ver alguna gotera y los asientos son incómodos. A esto sumamos que no es accesible para la personas con movilidad reducida, entre una larga lista de carencias. Hasta que llegue esa remodelación, la Concejalía de Cultura ha realizado una serie de mejoras en la iluminación y en el sonido del teatro.

La remodelación del jardín del Palacio Juan Pizarro de Aragón sí que ha supuesto una pérdida de su valor histórico, en tanto en cuanto, no se ha aprovechado ni reutilizado ningún elemento integrante del mismo. Aunque dado su lamentable estado de conservación era prácticamente imposible reci-

\footnotetext{
${ }_{9}^{9}$ Archivo Municipal de Trujillo, Zorita Carrero, J.R. (1986).
} 
clar sus elementos integrantes, por lo que la única actuación posible fue recrear su ubicación y forma. Sin duda, la intervención es innovadora pues prácticamente no se conservaba nada del antiguo jardín, pero no es del todo acorde con su entorno pues debía integrarse en el paisaje urbano de la localidad. Respecto a su funcionalidad, se ha recuperado el carácter recreativo del jardín decimonónico consiguiendo que se conciba como un espacio cultural destinado para la vía pública.

Fig. 7.- Teatro Gabriel y Galán de Trujillo (Cáceres) (Fotografía: Fernández Rojo, L.)

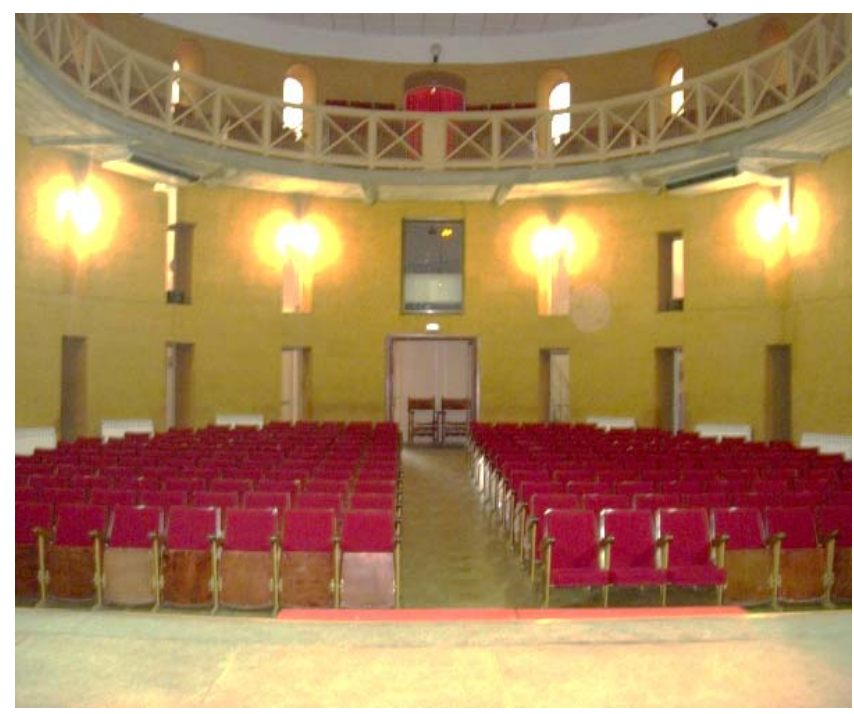

2. Conclusiones: líneas de conservación en la arquitectura para espectáculos.

En líneas generales contamos con escasos ejemplos de esta tipología arquitectónica porque fueron muy transformados, pues aunque fueran levantados como teatro, posteriormente se utilizarían como sala de baile, en los años cuarenta se convirtieron en cines, a finales del siglo $X X$ se rehabilitan como discoteca o $p u b$ o con suerte como multicines, y finalmente llegan al siglo XXI como piso, garaje o como un solar vacío.

En la actualidad se ha emprendido una tímida iniciativa restauradora para la revalorización de estos espacios, motivada más que nada por la necesidad de la actualización de estos escenarios, por la fascinación de los arquitectos en las posibilidades que brinda esta tipología arquitectónica. Pero estas propuestas finalmente han caído en manos de agentes de naturaleza comercial, sin una 
formación histórica-artística, que buscan que las renovaciones de estas salas no se sustenten en sus formas primitivas en aras de una transformación más radical que les permitan ofrecer diversos espectáculos y no solo el teatral o cinematográfico. Al prescindir de la necesaria asistencia de los especialistas con los conocimientos técnicos requeridos, muchas restauraciones se han limitado a dotar al establecimiento de una serie de soportes de espectáculos mínimos para garantizar la puesta en escena de las actuaciones, no justificándose de este modo la inversión realizada. No obstante las intervenciones han servido para prolongar la existencia de muchos teatros y cines, recuperando su presencia como sedes de una actividad social que estaba prácticamente extinguida.

Por supuesto que estos inmuebles deben actualizarse a las necesidades actuales y por ello deben ir cambiando a lo largo del tiempo, para que sigan funcionando. $\mathrm{Y}$ es que el teatro ya no es lo que era y sus representaciones son muy diferentes a las que se daban en el pasado siglo, por ello es fundamental adaptar el espacio escénico a las exigencias actuales de la función teatral y de los servicios que ésta precisa.

La tendencia actual es la recuperación de antiguos edificios tal y como eran en su origen para que puedan utilizarse, tesis que también es defendida por el arquitecto Felipe Delgado (autor de las restauraciones de los teatros María Guerrero y La Zarzuela de Madrid, y del Corral de Comedias de Almagro). El motivo es que no contamos con tanto patrimonio como para no intervenir aquellos edificios que tienen una cierta antigüedad. Pues si una sociedad es rica culturalmente, lo lógico es que construya un nuevo teatro en otro espacio y no destruya uno antiguo para edificar sobre él uno nuevo.

Tras la Guerra Civil, la mayoría de los teatros fueron transformados en cines, y en la actualidad no suelen recuperarse con su función primitiva. El motivo principal es la profunda crisis que vive la industria del cine y del teatro, rehabilitarlos con una sola función es condenarlos a la ruina por ello se optan por espacios multifuncionales para albergar diversas actividades culturales. Manteniéndose así su carácter cultural pero modificando su función en pos de su uso.

Respecto a sus intervenciones, no siguen ninguna teoría de la historia de la restauración, son actuaciones modernas que se concentran en el escenario para adecuarlo a sus necesidades para la representación teatral actual. Para que las torres escénicas puedan alojar la maquinaria se modifican los volú- 
menes del edificio original, convirtiéndose en la seña de esa modernización. Según Joan Font director del grupo teatral Comediants, los escenarios no permiten formas de representación que faciliten la ruptura de la convención del teatro a la italiana. Por ello muchos dramaturgos buscan espacios diferentes a los teatros para llevar a cabo representaciones innovadoras, lo que explicaría el desuso de muchos de estos inmuebles así como su rehabilitación con otras funciones por su inutilización.

En definitiva la preocupación actual por la recuperación de este tipo de arquitectura, es en este sentido síntoma de una nueva sensibilidad hacia estos inmuebles culturales.

Pero depende de quien la lleve a cabo, la intervención será respetuosa o radical. Si la rehabilitación es dirigida por un arquitecto y un equipo cualificado será respetuosa con el original, del edificio primitivo suele conservarse el exterior como una segunda piel que reviste la innovadora sala que para adaptarse al momento y necesidades actuales sufre sucesivas transformaciones ${ }^{10}$.

\section{Bibliografía.}

BARCO, E. (2011), Infraestructuras instalaciones Cine Municipal y Aledaños.

BARragán Del Puerto, N. (2009), Proyecto básico para la integración del museo de arte contemporáneo en el teatro Central Cinema, Azuaga.

De AReCHAGA, L. (1982); Proyecto de rehabilitación.

De ArechagA, L. (1982), Proyecto de restauración del Palacio La Merced en Llerena (Badajoz).

FERNÁNDEZ AlBA, A. L. (2008), "Arquitectura teatral (1950-2000)", RILCE: Revista de filología hispánica, 2002, Vol. 18, núm. 2. ADE teatro: Revista de la Asociación de Directores de Escena de España, 2008, núm. 123.

GRANADA HERNANDEZ PACHÓN (2012): Informe sobre la situación actual de la Iglesia de La Merced en Llerena (Badajoz).

KRAHE MARINA, E. (2005), Proyecto de construcción de Espacio Escénico en Zafra (Badajoz).

${ }^{10}$ Fernández Alba, A. L. (2008). 
Peralta Del Amo, M. (2007), Proyecto de ejecución para la rehabilitación del Antiguo Mercado de Abastos en Teatro. Navalmoral de la Mata. Cáceres.

TEIJEIRO FUENTES, J. (1998), Informe técnico sobre valoración arquitectónica de dos edificios propiedad de la sociedad privada Círculo Pacense.

REDONDO ROSARIO, A. y ARQUITES (2006), Primera separata para el proyecto de reforma del Cine San Vicente. Sustitución de cubierta de la zona de escenario y bar.

Zorita Carrero, J.R. (1986), Proyecto de Restauración y habilitación del Palacio de Juan Pizarro de Aragón para centro cultural. 